Main Outcome Measures Prevalence, among singleton pregnancies, of late miscarriage (20-23 weeks gestation), termination of pregnancy for fetal anomaly ( $\geq 20$ weeks gestation), stillbirth ( $\geq 24$ weeks gestation), fetal death (miscarriage or antepartum stillbirth), perinatal mortality, neonatal mortality, post-neonatal mortality, and all infant mortality. Crude and adjusted (for maternal age, gestational age, where appropriate, birth weight, standardised for sex and gestational age, ethnicity, cigarette smoking status, index of multiple deprivation, and sex of infant/fetus) ORs of each outcome among maternal obese (BMI $\geq 30 \mathrm{~kg} / \mathrm{m}^{2}$ ) compared to maternal recommended BMI (BMI 18.5-24.9 kg/m²).

Results Compared to women of recommended BMI, obese women were at significantly greater risk of a fetal death (adjusted odds ratio (aOR) 2.94 (95\% CI 2.02 to 4.27), $\mathrm{p}<0.001$ ), including late miscarriage (aOR 3.27 (95\% CI 1.43 to 7.44$), \mathrm{p}=0.005$ ) and antepartum stillbirth (aOR 2.86 (95\% CI 1.88 to 4.35$), \mathrm{p}<0.001)$, perinatal death (aOR 2.54 (95\% CI 1.70 to 3.79), p<0.001), including stillbirth (aOR 2.77 (95\% CI 1.86 to 4.13$), \mathrm{p}<0.001)$ and early neonatal death (aOR 3.00 (95\% CI 1.12 to 8.03), $\mathrm{p}=0.03$ ), and infant death (aOR 2.49 ( $95 \%$ CI 1.34 to 4.62 ), $\mathrm{p}=0.004$ ), including neonatal death (aOR 2.57 (95\% CI 1.13 to 5.88), $p=0.03$ ). There was no significant association between maternal obesity and post-neonatal death (aOR 2.27 (95\% CI 0.89 to 5.80), $\mathrm{p}=0.09$ ). The effect of obesity on risk of stillbirth was greater among small-for-gestational-age fetuses, but less among current smokers. Except for higher rates of pre-eclampsia among stillbirths, no specific cause of death could explain the increased odds of fetal and infant death among the obese.

Conclusion Early-pregnancy obesity is significantly associated with fetal and infant death, independent of the known relationship with congenital anomalies. Further studies are required to investigate the specific mechanisms involved. In the meantime, women should be made aware of these risks and supported to optimise their weight before pregnancy.

\section{P12 MATERNAL SMOKING DURING PREGNANCY AND OFFSPRING BONE MASS AT AGE 9 YEARS}

doi:10.1136/jech.2010.120477.12

${ }^{1} \mathrm{C}$ Macdonald-Wallis, ${ }^{2} \mathrm{~J} H$ Tobias, ${ }^{1} \mathrm{G}$ Davey Smith, ${ }^{1} \mathrm{D}$ A Lawlor. ${ }^{1} \mathrm{M} R \mathrm{C}$ Centre for Causal Analyses in Translational Epidemiology, Department of Social Medicine, University of Bristol, Bristol, UK; ${ }^{2}$ Academic Rheumatology, Clinical Science at North Bristol, University of Bristol, Bristol, UK

Objective Studies in neonates have demonstrated an adverse relationship between maternal smoking during pregnancy and fetal bone mineral accrual. We aimed to investigate an intrauterine influence of maternal smoking in pregnancy on offspring bone mass during childhood.

Design Prospective birth cohort study: the Avon Longitudinal Study of Parents and Children (ALSPAC).

Setting Mothers were resident in Avon, UK during pregnancy and had expected delivery dates between April 1991 and December 1992. Participants and Methods Our analysis included 7121 children attending a research clinic at mean age 9.9 years. We used multiple regression models to compare associations of maternal and paternal smoking at the time of pregnancy with measures of bone size and density in the children, since a greater maternal association would provide evidence of a direct intrauterine effect on the fetus. Multivariate multiple imputations were used to account for missing data. Main Outcome Measures DXA measured total body less head $(\mathrm{TBLH})$ and spine bone mineral content (BMC), bone area $(\mathrm{BA})$ and bone mineral density (BMD).

Results Maternal smoking in any trimester of pregnancy was associated with increased TBLH BMC, BA and BMD in girls (mean difference $(95 \% \mathrm{CI}$ ) (using sex-specific SD scores): 0.13 (0.05 to
0.22 ), 0.13 (0.04 to 0.21 ) and 0.13 (0.04 to 0.22 ), respectively) but not in boys $(0.01$ ( -0.07 to 0.09$), 0.00$ ( -0.08 to 0.08$), 0.04(-0.05$ to 0.12$)$ ) in confounder-adjusted models. It was also associated with spine BMC, BA and BMD in girls (0.13 (0.03 to 0.23), 0.12 (0.03 to $0.22), 0.10(0.00$ to 0.21$))$ but not in boys $(0.03$ ( -0.06 to 0.12$), 0.00$ $(-0.09$ to 0.09$), 0.05$ ( -0.04 to 0.14$)$ ). Paternal smoking associations were similar, with no statistical evidence for a difference between maternal and paternal associations (all $p$ values $>0.15$ ). Maternal associations increased on adjustment for offspring birth weight and gestational age but attenuated to the null on adjustment for the child's height and weight at age 9 years. Further investigation indicated that these relationships were driven mainly by offspring weight rather than height.

Conclusions There was little evidence of a relationship between maternal smoking during pregnancy and bone mass in boys. Our parental comparisons and multivariable analyses suggested that the positive associations between maternal smoking and bone mass in girls were likely to be attributable to shared familial characteristics related to offspring adiposity rather than an intrauterine mechanism.

\section{P13 ASSOCIATION OF PRENATAL AND POSTNATAL SMOKING AND ALCOHOL CONSUMPTION ON BIRTH WEIGHT IN THE WHITE BRITISH POPULATION IN BRADFORD: PRELIMINARY FINDINGS FROM THE BORN IN BRADFORD STUDY}

doi:10.1136/jech.2010.120477.13

${ }^{1,2}$ E Petherick, ${ }^{2} \mathrm{R}$ Parslow, ${ }^{2} \mathrm{P}$ McKinney, ${ }^{1} \mathrm{D}$ Tufnell, ${ }^{3} \mathrm{D}$ Leon, ${ }^{1}$ Pauline Raynor, ${ }^{4} \mathrm{D}$ Lawlor, ${ }^{1} \mathrm{~J}$ Wright. ${ }^{1}$ Bradford Institute for Health Research, Bradford Royal Infirmary, Bradford, UK; ${ }^{2}$ Paediatric Epidemiology Group, University of Leeds, Leeds, UK; ${ }^{3}$ London School of Hygiene \& Tropical Medicine, London, UK; ${ }^{4}$ MRC Centre for Causal Analyses in Translational Epidemiology, Department of Social Medicine, University of Bristol, Bristol, UK

Background Exposure to cigarette smoke and heavy alcohol consumption has been known to influence birth weight and risk of being small for gestational age. Despite this knowledge, very little exploration of the timing of cigarette smoking and alcohol exposure on growth has been undertaken.

Aims To investigate the effect of dose and timing of pre-pregnancy and prenatal alcohol and cigarette exposure on birth weight.

Methods The Born in Bradford cohort includes women of diverse ethnicities, with $46 \%$ of the population being of South Asian origin. However, smoking prevalence $(5.9 \%)$ and alcohol consumption rates $(0.4 \%)$ in pregnant women of South Asian origin were too low for meaningful analyses. Therefore, analyses were restricted to the white British women who had completed a questionnaire and had a live singleton full-term birth at the Bradford Royal Infirmary, between October 2007 and August 2009. Data were available on smoking and alcohol consumption at three different time points: in the 3 months prior to pregnancy, first 3 months of pregnancy and when the baseline questionnaire was administered (26-28 weeks gestation) by trained interviewers. This information was used to classify women into three mutually exclusive categories separately for both smoking and alcohol: smoker/drinker at all time points, decreasing or quitting at different time periods throughout and nonsmokers/drinkers at all time points. Mean differences in birth weight between these categories were investigated using multivariable linear regression. The model adjusted for maternal age, baby's gender, parity, area deprivation, caffeine consumption, and mother's weight at her first antenatal clinic appointment.

Results 1933 white British mothers and their children were eligible for inclusion. $39.7 \%$ were regular smokers in the 3 months prior to pregnancy and $33.6 \%$ during pregnancy, whilst $72 \%$ consumed alcohol in the 3 months prior to pregnancy and $40 \%$ during 
pregnancy. $29.3 \%$ of pregnant women both smoked and drank before pregnancy reducing to $15.1 \%$ during pregnancy. Adjusted analysis showed that birth weight was negatively associated with continual smoking, $-231 \mathrm{~g}$ ( $95 \%$ CI -318.3 to -144.5$)$, whilst quitting or decreasing smoking was associated with $-68.5 \mathrm{~g}$ reduction ( $95 \%$ CI -119.7 to -17.4 ) compared to nonsmokers. No association with birth weight was observed for alcohol consumption $(-0.16 \mathrm{~g}, 95 \% \mathrm{CI}-69.9$ to 69.6$)$.

Conclusions Timing of quit smoking interventions should concentrate on the pre-pregnancy period to ensure optimal benefits on birth weight. These results suggest that smoking cessation advice provided in the ante natal period may have limited benefit.

\section{P14 INTERVENTIONS TO INCREASE THE EARLY INITIATION OF ANTENATAL CARE IN SOCIALLY DISADVANTAGED AND VULNERABLE WOMEN: A SYSTEMATIC REVIEW}

doi:10.1136/jech.2010.120477.14

L Oakley, R Gray, J J Kurinczuk, P Brocklehurst, J Hollowell. National Perinatal Epidemiology Unit, University of Oxford, Oxford, UK

Objective To systematically evaluate the effectiveness of interventions to increase the early initiation of comprehensive antenatal care in socially disadvantaged and vulnerable women.

Design Systematic review.

Data Sources Major bibliographic databases (Medline, Cinahl, Embase, PsycINFO, HMIC, CENTRAL) and other online libraries and resources were searched to identify relevant English language journal articles published 1990-2009. We included comparative studies (experimental or observational) evaluating the effectiveness of an intervention on the proportion of women initiating antenatal care by any defined cut-off point $\leq 20$ weeks in a disadvantaged or vulnerable population. In order to focus on interventions relevant in the context of the UK National Health Service, we excluded studies from low-income countries and those relating to financial interventions such as extension of health insurance coverage or similar. Review Methods Two reviewers independently extracted data for eligible studies; assessed internal validity using the GATE checklist and considered whether the studies provided evidence of a beneficial effect.

Results Over 3000 citations were screened. Sixteen eligible studies were identified; 14 conducted in the USA, one in Australia and one in the UK. All were observational evaluations. Twelve studies evaluated interventions targeted at specific disadvantaged or vulnerable subgroups of the population (predominantly ethnic minority women or teenagers); the remaining studies evaluated interventions in more generally socioeconomically disadvantaged populations. Eleven studies evaluated interventions that involved outreach or other community-based services, and five evaluated interventions that involved alternative models of clinic-based antenatal care. Overall, the quality of evidence was poor. Only one study, which evaluated a US home visiting intervention delivered by paraprofessional women to pregnant adolescents, was considered to have adequate internal validity. The reviewers considered the evidence relating to this intervention to be inconclusive but consistent with a possible beneficial effect of the intervention on timing of initiation of antenatal care.

Conclusion We found insufficient evidence of adequate quality to reliably conclude that any of the interventions considered were effective at increasing the early initiation of antenatal care in socially disadvantaged and vulnerable women. There was weak evidence of effectiveness relating to one intervention based on home visiting for pregnant adolescents. Findings were inconclusive for all other included interventions, although we identified several strategies that might warrant further consideration and possibly more robust evaluation. The results of this review highlight the paucity of evidence and the need for further high quality research to ensure that future service innovations are evidence based.

\section{P15 EFFECT OF TRAINING DOCTORS IN COMMUNICATION SKILLS ON SYRIAN WOMEN'S SATISFACTION DURING LABOUR: A STEPPED WEDGE CLUSTER RANDOMISE TRIAL}

doi:10.1136/jech.2010.120477.15

${ }^{1} \mathrm{M}$ Kanaan, ${ }^{2} \mathrm{H}$ Bashour, ${ }^{2} \mathrm{~A}$ Abdulsalam, ${ }^{2} \mathrm{M}$ Kharouf, ${ }^{2} \mathrm{~S}$ Cheikha, ${ }^{2} \mathrm{M}$ Tabbaa. ${ }^{1}$ Department of Health Sciences, University of York, York, UK; ${ }^{2}$ Faculty of Medicine, Damascus University, Damascus, Syria

Objective To test the effect of training residents in interpersonal and communication skills on women's satisfaction with patient-doctor relationship in labour and delivery rooms.

Design A stepped-wedge cluster randomised trial.

Setting Maternity wards in four teaching hospitals in Damascus and homes of participating women.

Participants Women delivering in the hospitals under study, via vaginal delivery with living baby, who consent to participate in the study. Difficult labour and high-risk pregnancies are excluded. Residents working in the study hospitals during the implementation phase who agree to take part in the study.

Main Outcome Measure Women's satisfaction with interpersonal relationships in labour and delivery rooms measured via a series of questions on a Likert scale and based on the Medical Interview Satisfaction Scale.

Results Women were on average 25 years old, 95\% were homemakers, $76 \%$ had primary education, $48 \%$ lived in shared accommodation and $26 \%$ were nulliparous. At the individual level, the mean score of overall satisfaction of women was $68.66(\mathrm{SD}=14.24)$ out of a possible score of a 100 in the control group and 70.79 $(\mathrm{SD}=13)$ in the treatment group. At the hospital level, the mean scores of overall satisfaction of women were $70(\mathrm{SD}=4.70)$ and 70.99 $(\mathrm{SD}=4.85)$ for the control and treatment group, respectively. Using (generalised) linear mixed models approach to account for the study design, we were not able to detect a difference between the treatment and control group on the overall satisfaction of women.

Conclusion The training package does not seem to be associated with higher overall satisfaction scores.

\section{P16 QUANTIFYING THE RISK OF DEPRIVATION ON PRETERM BIRTH IN UK MATERNITY UNITS}

doi:10.1136/jech.2010.120477.16

${ }^{1} \mathrm{D}$ Taylor-Robinson, ${ }^{2} \mathrm{U}$ Agarwal, ${ }^{1} \mathrm{M} \mathrm{J}$ Platt, ${ }^{3} \mathrm{P}$ Diggle, ${ }^{4} \mathrm{~B}$ Yoxall, ${ }^{5} \mathrm{Z}$ Alfirevic. ${ }^{1}$ University of Liverpool, Liverpool, UK; ${ }^{2}$ Liverpool Women's NHS Foundation Trust, Liverpool, UK; ${ }^{3}$ CHICAS, Lancaster University, Lancaster, UK; ${ }^{4}$ Women's NHS Foundation Trust, Liverpool, UK; ${ }^{5}$ Fetal and Maternal Medicine, University of Liverpool, Liverpool, UK

Objective To explore risk factors for preterm birth (PTB) between 24 +0 and $34+0$ gestational weeks in the UK's largest maternity unit, with a particular focus on low risk pregnancies and the effect of socioeconomic status.

Design Retrospective cohort study of routinely collected obstetric and neonatal data.

Setting 50486 singleton pregnancies booked at the Liverpool Women's NHS Foundation Trust for all women delivering after 24 +0 weeks gestation over a 7-year period from 2002 to to 2008 .

Main Outcome Measure The primary outcome was preterm birth. Pregnancies were stratified into three groups: low risk; those complicated by medical problems; pregnancies in women with a 